\title{
Letter: Assessment of aortic valve stenosis severity using intelligent phonocardiography
}

Arash Gharehbaghi, Inger Ekman, Per Ask, Eva Nylander and Birgitta Janerot-Sjoberg

\author{
Linköping University Post Print
}

\section{Tweet}

N.B.: When citing this work, cite the original article.

Original Publication:

Arash Gharehbaghi, Inger Ekman, Per Ask, Eva Nylander and Birgitta Janerot-Sjoberg, Letter: Assessment of aortic valve stenosis severity using intelligent phonocardiography in INTERNATIONAL JOURNAL OF CARDIOLOGY, 2015, International Journal of Cardiology, (198), 58-60.

http://dx.doi.org/10.1016/j.ijcard.2015.06.126

Copyright: Elsevier

http://www.elsevier.com/

Postprint available at: Linköping University Electronic Press

http://urn.kb.se/resolve?urn=urn:nbn:se:liu:diva-121420 


\section{Assessment of aortic valve stenosis severity using intelligent phonocardiography}

Arash Gharehbaghi ${ }^{\mathrm{a},}{ }^{*}, 1$, Inger Ekman $^{\mathrm{b}}$, Per Ask $^{\mathrm{a}}$, Eva Nylander $^{\mathrm{b}, \mathrm{c}}$, Birgitta Janerot-Sjoberg ${ }^{\mathrm{d}, \mathrm{e}, \mathrm{f}}$

${ }^{\text {a }}$ Physiological Measurements, Department of Biomedical Engineering, Linköping University, Linköping, Sweden.

${ }^{\mathrm{b}}$ Department of Clinical Physiology, Department of Medical and Health Sciences, Faculty of Health Sciences, Linköping University, Linköping, Sweden.

${ }^{\mathrm{c}}$ Center for Medical Image Science and Visualization, Linköping University, Linköping, Sweden.

${ }^{\mathrm{d}}$ Division of Medical Imaging and Technology, Department of Clinical Science, Intervention and Technology, Karolinska Institute, Stockholm, Sweden.

${ }^{\mathrm{e}}$ Departments of Clinical Physiology and Medical Technology, Karolinska University Hospital, Stockholm, Sweden.

${ }^{\mathrm{f}}$ School of Technology and Health, KTH Royal Institute of Technology, Stockholm, Sweden.

\section{* Corresponding address:}

Physiological Measurements, Department of Biomedical Engineering, Linköping University, Linköping, Sweden.

Tel.: +46 736256654

Email: arash.ghareh.baghi@liu.se

\footnotetext{
${ }^{1}$ Present address: Division of Intelligent Future Technology, Department of Innovation, Design and Technology, Mälardalen University, Västerås, Sweden.

Email: arash.ghareh.baghi@mdh.se
} 


\section{Letter to the Editor}

Valvular aortic stenosis (AS) is the third most prevalent cardiovascular disease in developed countries, reported in $2.8 \%$ of patients $>75$ years-of-age [1-3]. Echocardiography is the gold standard diagnostic tool, recommended by both the American Heart Association (AHA) and the European Society of Cardiology (ESC) [4-5]. According to the directives of the European and American Echocardiographic Societies (EAE/ASE), the severity of AS is essentially based on three parameters; peak systolic velocity (PSV), mean pressure gradient (MPG), and the calculated aortic valve area (AVA) [6]. The skill, experience and knowledge of the operator play key roles in the accuracy of assessments. For elderly comorbidity, and in rural areas, access to echocardiography can be a problem. It is therefore desirable to find a frugal and reliable screening tool to separate innocent murmur or mild AS from significant AS in primary healthcare centers. The present study describes the application of the intelligent phonocardiography (IPCG) for grading AS severity using the phonocardiographic (PCG) signal. Both reliability and reproducibility were statistically evaluated using echocardiographic classification as gold standard.

In- and outpatient echocardiographic referrals to the Linköping University Hospital Heart Center, where patients diagnosed with AS of varying severity as the predominate finding, were included in the study. After obtaining the regional ethical approval which is in compliance with the Declaration of Helsinki, fifty patients gave their informed consent to participate.

The blood flow time-velocity profiles were obtained in the left ventricular outflow tract (LVOT) and at the aortic orifice using pulsed and continuous-wave Doppler, respectively. PSV, MPG, velocity time integrals (VTI) and the LVOT diameter were measured and the effective AVA was calculated during the ejection time period (ETP), using the continuity equation [6]. The degree of AS was graded mild, moderate or severe, based on the AVA, MPG and PSV, according to the recommendation of the ESC 
[5]. The left ventricular (LV) systolic function was visually judged semi-quantitatively as normal (0), mildly (1), moderately (2) or severely (3) reduced, based on the ejection fraction (EF) [7].

The degree of mitral regurgitation was estimated using a 4-degree scale; no or minimal (1), mild (2), moderate (3) or severe (4) regurgitation. The study population is listed in Table 1.

\section{Table 1}

An Elite ${ }^{\mathrm{TM}}$ electronic stethoscope (WelchAllyn, USA) was used for signal acquisition together with a personal computer (DELL, USA) equipped with a 16-bit sound card. For each patient, four PCG signals of 10-second durations were thoracically recorded in the supine position; two from the apex and two from the aortic area (right parasternal second intercostal space).

The systolic murmur was analyzed in order to grade the severity of AS, by applying a novel processing algorithm, where the wavelet transformation technique served as a mathematical tool, exploring the joint time-frequency content of the AS murmur. The energy content of the wavelet transform were calculated over a set of overlapping windows and used in multiple regression analysis. The optimal windows characteristics along with the wavelet specifications were statistically obtained by using the forwardbackward stepwise regression analysis in which five echocardiographic parameters acted as predictors; AVA, MPG, PSV, VTI and ETP. The predicted values were employed by an artificial neural network for grading the AS severity into one of the three levels; mild, moderate and severe.

Performance of the IPCG was statistically validated using its accuracy as performance measure, defined as the number of correctly assessed patients as a function of the total number of patients. For reliability validation, the accuracy of the algorithm was calculated by randomly selecting $70 \%$ of the PCG recordings (only one recording from each patient) for training and the rest for accuracy calculation. The 
same procedure was repeated several times with different random sets of training data, all having the same size. Confidence interval of the calculated accuracies was an indication of the IPCG reliability. In order to validate reproducibility of the IPCG, $70 \%$ of the patients were randomly selected for validation. One recording from each patient was selected for training and the other recordings for accuracy calculation. The reproducibility was investigated based on the confidence interval of the calculated accuracies.

The results of the regression analysis for AVA, MGP, PSV, VTI and ETP are demonstrated in Figure1.

\section{Figure 1}

The measured and predicted values correspond to the echocardiographic findings and the estimated values, respectively. A relevant regression with an appropriate goodness-of-fit $(r>0.8)$ was observed for all of the five echocardiographic parameters.

Reliability and reproducibility of the IPCG were estimated to be 80-83 (SD 6.7) \% and 90-93 (SD 6.8) $\%$, respectively, when applying the double-sided t-test on a $95 \%$ confidence level. In the severity assessment, a perfect match was seen between the two techniques in all but five patients, where the assessment deviated one degree only.

In conclusion the IPCG shows potential to be employed as an efficient decision support tool in primary healthcare centers, and may thereby contribute to superior prioritization of more expensive, less available echocardiographic examinations. The processing algorithm employed in this study involves modest complexity in implementation when it comes to a required expanded test phase, even allowing incorporation into an android-based graphical user interface that can be used in a home-based healthcare system. 
This study was supported by the Swedish Research Council, the NovaMedTech EU structural foundation program at Linköping University, the Swedish Heart Lung Foundation, and strategic medical technology grants from the Karolinska Institute.

\section{References}

[1] N. Saikrishnan, G Kumar, F. J. Sawaya, S. Lerakis, A. P. Yoganathan. Accurate assessment of Aortic Stenosis: A Review of Diagnostic Modalities and Hemodynamics. Circulation 2014; 129:244-253.

[2] B. A. Carabello. Clinical practice: Aortic stenosis. N. Engl. J. Med. 2002; 346:677-682.

[3] V. T. Nkomo, J. M. Gardin, T. N. Skelton, J. S. Gottdiener, C. G. Scott, M. Enriquez-Sarano. Burden of valvular heart diseases: a population-based study. The Lancet 2006; 368:1005-1011.

[4] D. C. Goff et al. 2013 ACC/AHA guideline on the assessment of cardiovascular risk: a report of the American College of Cardiology/American Heart Association Task Force on Practice Guidelines. J Am. Coll. Cardiol. 2014 Jul 1;63(25 Pt B):2935-59.

[5] A. Vahanian et al. Guidelines on the management of valvular heart disease (version 2012): The Joint Task Force on the Management of Valvular Heart Disease of the European Society of Cardiology (ESC) and the European Association for Cardio-Thoracic Surgery (EACTS). Eur. Heart J. (2012) 33 (19): 2451-2496.

[6] H. Baumgartner, J. Hung, J. Bermejo et al. Echocardiographic assessment of valve stenosis: EAE/ASE recommendations for clinical practice. Eur. J. Echocardiogr. 2009; 10:1-25.

[7] R. M. Lang, M. Bierig, R. B. Devereux, et al. Recommendations for chamber quantification. Eur. J. Echocardiogr. 2006; 7:79-108. 


\section{Table 1}

The patients' demography characteristics. AS severity was assessed by echocardiography..

\begin{tabular}{|lccc|c|}
\hline & Mild AS & Moderate AS & Severe AS & Total \\
& $\mathrm{N}=10(20 \%)$ & $\mathrm{N}=14(28 \%)$ & $\mathrm{N}=26(52 \%)$ & $\mathrm{N}=50$ \\
\hline Average height $(\mathrm{cm}) \pm \mathrm{SD}$ & $171.40 \pm 8.87$ & $167.86 \pm 7.91$ & $166.77 \pm 9.42$ & $168.00 \pm 8.92$ \\
Average weight $(\mathrm{kg}) \pm \mathrm{SD}$ & $72.50 \pm 16.26$ & $75.43 \pm 15.75$ & $76.42 \pm 15.39$ & $75.36 \pm 15.41$ \\
Average body surface area $\left(\mathrm{m}^{2}\right) \pm \mathrm{SD}$ & $1.87 \pm 0.20$ & $1.85 \pm 0.20$ & $1.85 \pm 0.22$ & $1.85 \pm 0.21$ \\
Average body mass index $\left(\mathrm{kg} / \mathrm{m}^{2}\right) \pm \mathrm{SD}$ & $25.59 \pm 4.01$ & $26.69 \pm 4.56$ & $27.33 \pm 4.26$ & $26.80 \pm 4.26$ \\
Normal left ventricle function & $8(16 \%)$ & $14(28 \%)$ & $21(42 \%)$ & $43(86 \%)$ \\
Mild left ventricle dysfunction & $2(4 \%)$ & 0 & $2(4 \%)$ & $4(8 \%)$ \\
Moderate left ventricle dysfunction & 0 & 0 & $3(6 \%)$ & $3(6 \%)$ \\
Severe left ventricle dysfunction & 0 & 0 & 0 & 0 \\
Minimal mitral regurgitation & $8(16 \%)$ & $11(22 \%)$ & $9(18 \%)$ & $33(66 \%)$ \\
Mild mitral regurgitation & $2(4 \%)$ & $3(6 \%)$ & $3(6 \%)$ & $14(28 \%)$ \\
Moderate mitral regurgitation & 0 & 0 & 0 & $3(6 \%)$ \\
Severe mitral regurgitation & 0 & 0 & 0 \\
\hline
\end{tabular}




\section{Figure caption}
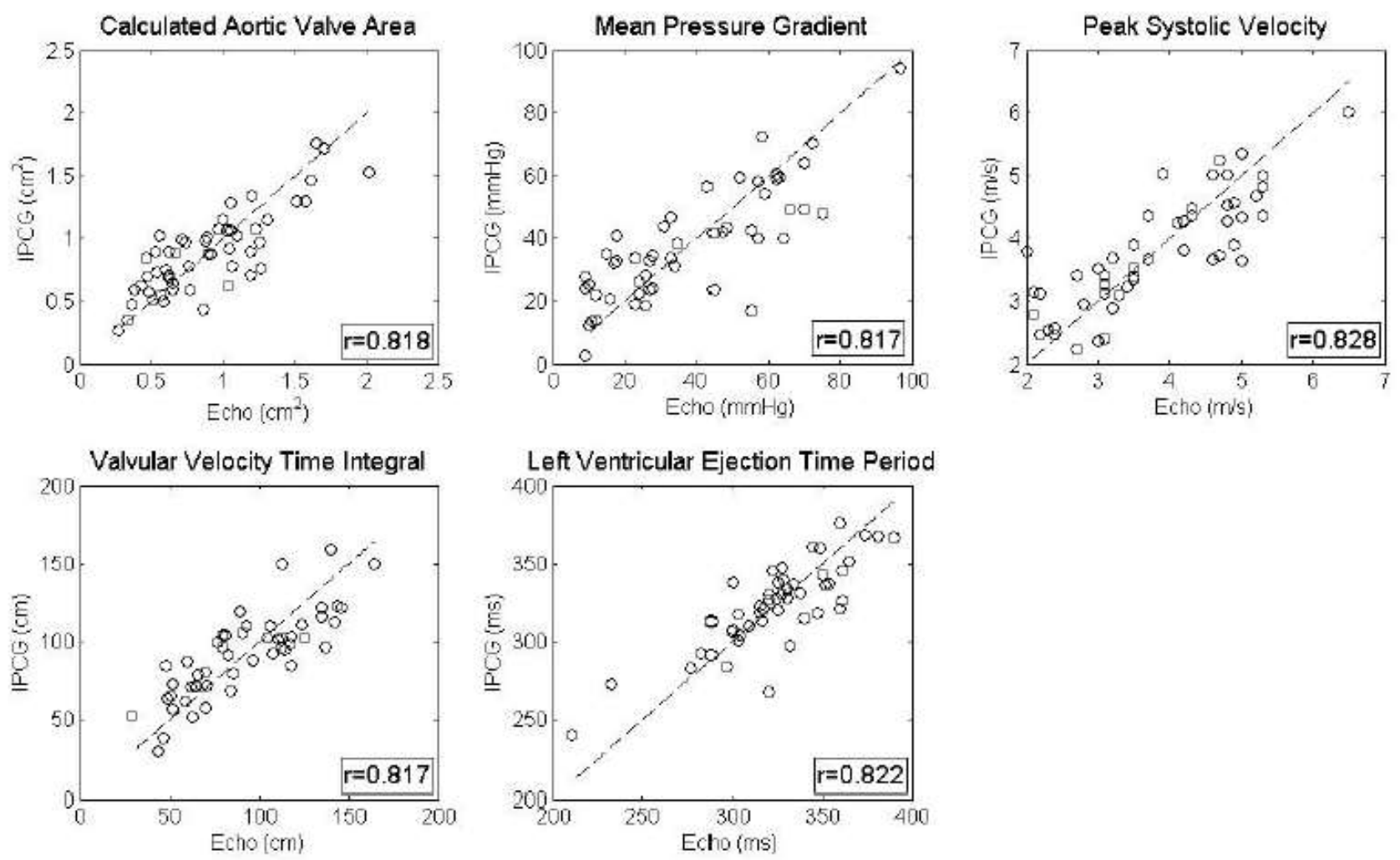

Figure 1 Results of the regression analysis in predicting the echocardiographic findings from the heart murmurs. The $r$ represents goodness-of-fit resulting from the regression analysis. The IPCG and Echo correspond to the values obtained by the intelligent phonocardiography and echocardiography, respectively. No value significantly departs from the line of unity. 